\title{
Lemierre syndrome
}

\author{
Ajay Malhotra $\cdot$ Per-Lennart Westesson
}

Received: 4 July 2009 /Revised: 13 September 2009 /Accepted: 16 October 2009 / Published online: 8 December 2009

(C) Springer-Verlag 2009

A previously healthy 16-year-old girl with a 5-day history of sore throat and fever presented with shortness of breath and negative rapid strep and monospot tests. CT chest without IV contrast agent showed multiple bilateral pulmonary nodules in predominantly peripheral distribution (Fig. 1). CT neck with IV contrast agent demonstrated filling defect in a small tributary extending into the left internal jugular vein consistent with thrombus (Fig. 2). There was no evidence of oropharyngeal abscess clinically or on imaging. Initial blood cultures grew gram-negative anaerobic bacilli-fusobacterium species.

Lemierre syndrome is usually caused by an acute oropharyngeal infection in previously healthy young adults or

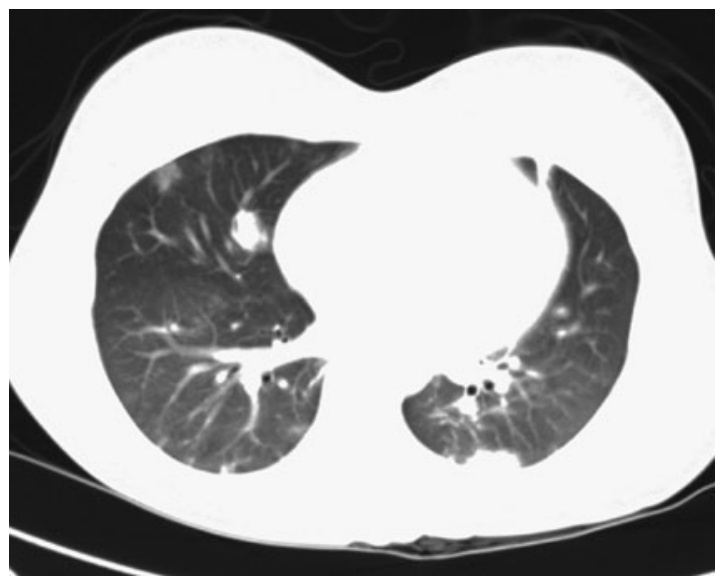

Fig. 1 CT chest

\footnotetext{
A. Malhotra $(\bowtie) \cdot$ P.-L. Westesson

Department of Radiology, Division of Neuroradiology, University of Rochester Medical Center,

P.O. Box 648, 601 Elmwood Ave.,

Rochester, NY 14642, USA

e-mail: ajayradiology@yahoo.com

\section{A. Malhotra}

Department of Radiology, Division of Neuroradiology,

Yale School of Medicine,

New Haven, CT, USA
}

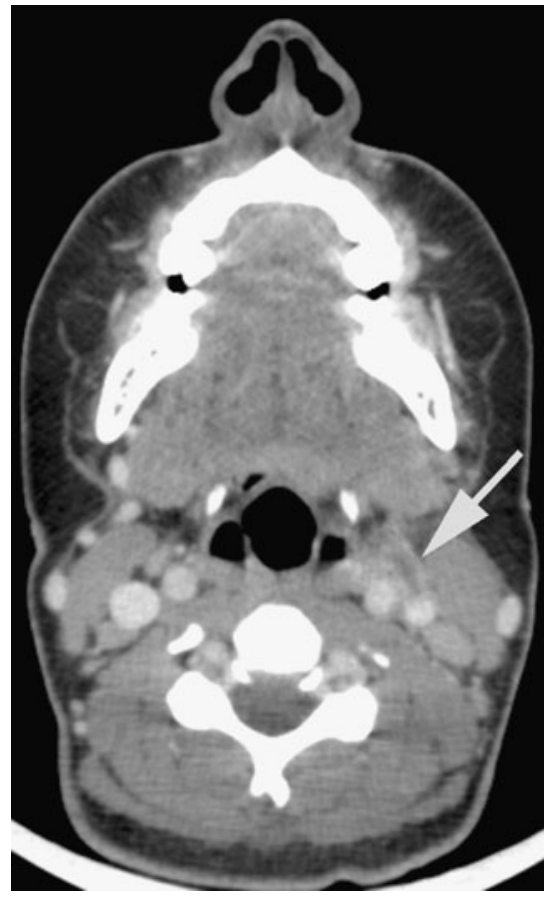

Fig. 2 CT neck with IV contrast agent

children resulting in septic thrombophlebitis of the internal jugular vein. Bacteraemia and septic emboli are a common sequela of internal jugular vein thrombosis, and the lungs are the most frequent target organs [1]. Fusobacterium necrophorum, a commensal in the oropharynx, is the usual causative organism. The disease is potentially lethal and early diagnosis and treatment with intravenous antibiotics is critical. The role of anticoagulation has remained controversial [2].

\section{References}

1. Lemierre A (1936) On certain septicemias due to anaerobic organisms. Lancet 1:701-703

2. Goldenberg NA, Knapp-Clevenger R, Hays T et al (2005) Lemierre and Lemierre-like syndromes in children: survival and thromboembolic outcomes. Pediatrics 116:e543-e548 